\title{
Subcutaneous Implantable Cardioverter Defibrillators Implantation Without Defibrillation Threshold Testing: A Single Center Experience
}

\author{
Bandar Al-Ghamdi a, c, d, Azam Shafquata, c, Nadiah Alruwailia , Shisamma Emmanuala , \\ Mohamed Shoukrib, c, Yaseen Mallawi ${ }^{\mathrm{a}}$
}

\begin{abstract}
Background: Subcutaneous implantable cardioverter defibrillator (S-ICD) system has been proven to be an effective therapy for prevention of sudden cardiac death $(\mathrm{SCD})$ in selected patients. Although the Shockless IMPLant Evaluation (SIMPLE) trial has shown that defibrillation threshold (DFT) testing is not necessary for transvenous ICD (TV-ICD) systems, it is still recommended for S-ICD systems. We aimed to study the efficacy and safety of S-ICD implantation without DFT in our Heart Center with the comparison of S-ICD patients' outcome to those with a single chamber TV-ICD without DFT in the same period.
\end{abstract}

Methods: A retrospective analysis of patients underwent S-ICD without DFT from December 2014 to May 2016 with the comparison to single chamber TV-ICD patients implanted during the same period.

Results: Thirty consecutive patients (23 males (76.7\%); mean age 41 \pm 13 years; mean left ventricular ejection fraction $30 \pm 12 \%$ ) received a S-ICD for primary (25 patients, $83.3 \%$ ) or secondary prevention (five patients, $16.7 \%$ ) of SCD. During a mean follow-up of $710.6 \pm 190$ days, three patients received 38 appropriate ICD shocks (90.5\%), and two patients received four inappropriate shocks $(9.5 \%)$. There were two mortalities (6.7\%): one cardiac and one non-cardiac. When compared to 30 consecutive who received a single chamber TV-ICD during the same period, there was no significant difference in mortality.

Conclusions: Implantation of S-ICD using intermuscular approach without DFT seems to be safe and effective. Data from large S-ICD registries with long-term follow-up, and preferably randomized controlled studies, are needed to confirm this finding.

Manuscript submitted November 20, 2017, accepted December 1, 2017

${ }^{a}$ Heart Center, King Faisal Specialist Hospital \& Research Centre, Zahrawi St, A1 Maather, Riyadh 12713, Saudi Arabia

${ }^{\mathrm{b} C e l l ~ B i o l o g y}$ Department, King Faisal Specialist Hospital \& Research Centre, Zahrawi St, Al Maather, Riyadh 12713, Saudi Arabia

${ }^{c}$ Alfaisal University, Riyadh, Saudi Arabia

${ }^{\mathrm{d} C}$ Corresponding Author: Bandar Al-Ghamdi, Heart Center, King Faisal Specialist Hospital \& Research Centre, Zahrawi St, Al Maather, Riyadh 12713, Saudi Arabia. Email: balghamdi@kfshrc.edu.sa

doi: https://doi.org/10.14740/cr638w
Keywords: Subcutaneous implantable cardioverter defibrillators; Defibrillation threshold testing; Shocks; Complications; Transvenous implantable cardioverter defibrillator

\section{Introduction}

Sudden cardiac death (SCD) is a leading cause of death worldwide. The introduction of the implantable cardioverter defibrillator (ICD) into clinical practice over the past 25 years has provided a life-saving therapy for primary and secondary prevention of SCD [1-4]. The subcutaneous ICD (S-ICD) is a relatively new technology that does not require vascular access or permanent intravascular defibrillator leads. It is developed to overcome the limitations and complications associated with traditional transvenous ICDs (TV-ICDs) such as venous thrombosis, cardiac perforation, lead fracture, and lead-related infective endocarditis. The S-ICD is approved for primary and secondary prevention of SCD based on clinical studies that demonstrated successful conversion of ventricular tachyarrhythmias with acceptably low rates of complications [5-7]. It is recommended for individuals who meet conventional ICD implantation criteria but do not have an indication for permanent pacing or cardiac resynchronization therapy, and not known to have recurrent ventricular tachycardia (VT) with expected good response to antitachycardia pacing (ATP), or preexisting unipolar pacemaker leads [7].

The term defibrillation threshold (DFT) testing refers to the minimum shock strength that defibrillates the heart [8]. A threshold below a specific value has been used as an acceptable criterion for device implantation $[9,10]$. The clinical measurement of DFT has only fair reproducibility and represents an estimate of a point on the patient's defibrillation probability of success curve. A variety of methods have been used to determine DFT [11]. With the improved technology available in current ICD devices, the need for DFT has come under question [12].

The potential benefits of DFT include confirmation of system integrity and reliable sensing, discovery of high DFTs needing system modification, increased assurance that successful defibrillation of VF should occur after the patient is discharged, allow programming lower first shock energies, and guarantee of a safety margin for testing after addition of medications that may increase DFT, such as amiodarone [12]. 
On the other hand, there are potential adverse effects or procedural complications related to DFT. The risks of DFT at the time of implantation could lead to a circulatory arrest and hypoperfusion related to VF itself. Other risks may also be related to the shocks alone or to the anesthetic drugs that are required for DFT. In one study, it was estimated that three of $19,067(0.016 \%)$ deaths were related to DFT testing [13].

The use of S-ICD is rapidly increasing in the United States, and the early adoption of this technology has been associated with a low complication rate and high rate of successful DFT testing despite use in patients with a high number of comorbidities [14]. Compared to TV-ICD, early S-ICD use was associated with a high rate of successful defibrillation for ventricular fibrillation (VF) during DFT testing [14]. Among 2,791 patients with S-ICD who underwent DFT testing, 2,588 (92.7\%), 2,629 (94.2\%), 2,635 (94.4\%), and 2,784 (99.7\%) were successfully defibrillated with $\leq 65, \leq 70, \leq 75$, and $\leq 80$ Joule (J), respectively [14].

In a matched cohort of S-ICD and TV-ICD patients (mostly dual-chamber devices), complication rates were similar. However, the S-ICD effectively reduced lead-related complications at the cost of non-lead-related complications. Both appropriate and inappropriate shock rates were similar [15]. The results of Shockless IMPLant Evaluation (SIMPLE) trial, which is a single-blinded, randomized, multicenter non-inferiority trial, showed that routine DFT at the time of TV-ICD implantation did not improve shock efficacy or reduce arrhythmic death [10]. This is not the case with S-ICD [16]. As a matter of fact, in a recent study looking at intraoperative DFT of S-ICD systems in 98 patients with the first shock of $65 \mathrm{~J}$, the intraoperative DFT was successful in 74 patients $(75 \%)$, successful test with reversed shock polarity in $14(15 \%)$, successful test after lead repositioning in one (1\%), successful test after pulse generator repositioning in five $(5 \%)$, and safety margin $<10 \mathrm{~J}$ in four (4\%) [17].

Current guidelines recommend DFT at the time of S-ICD implantation, based on the absence of evidence suggesting that it is safe to forgo DFT testing and concerns regarding increased defibrillation energy requirements compared to TV-ICDs [18].

To our knowledge, there are currently no data describing the use of the S-ICD without DFT, so we sought to 1) describe our experience with S-ICD implantation without DFT with evaluation of safety and efficacy of this approach and 2) compare outcomes of these patients to the outcomes of similar patients who received a traditional TV-ICD without DFT during the same period.

\section{Materials and Methods}

\section{Study population}

The data of 30 consecutive patients who underwent S-ICD systems implantation without DFT between December 2014 and May 2016, in the Heart Center at King Faisal Specialist Hospital \& Research Center, were retrospectively analyzed. The DFT was not performed due to high risk at the time of implantation with severe comorbidities in five patients or patient's request not have DFT when the treating physician felt that S-
ICD was the best option for the patient, e.g. patients with endstage renal disease (ESRD) or congenital heart disease in the other patients. The corresponding group consists of 30 patients implanted with a single chamber TV-ICD implantation without DFT during the same period.

\section{Data collection}

Data collection was performed at regular intervals by reviewing medical records for baseline characteristics, implantation data, and follow-up data on clinical outcomes, complications, and device therapy delivery. The survival status of patients was retrieved from electronic records.

\section{Definitions}

Primary prevention of SCD refers to use of ICDs in individuals who are at risk for, but have not yet had, an episode of sustained VT, VF, or cardiac arrest.

Secondary prevention refers to an indication for an ICD exclusively for patients who have survived one or more cardiac arrests or sustained VT [19].

\section{Three-incision technique for inserting S-ICD}

The conventional S-ICD implantation technique involves electrode and device implantation by three incisions - one for the lateral pocket and two parasternal incisions [20].

\section{Two-incision technique}

The parasternal part of the S-ICD electrode is positioned using a standard 11-Fr peel-away sheath avoiding the superior parasternal incision [21].

The generator-related complication was generator malfunction.

The lead-related complications included the following situations: dislodgement defined as X-ray-confirmed dislodgement of the lead combined with significant changes in sensing/ pacing performance, failure to capture at practical device output with no visible change in the lead position or considerable impedance rise, loose set screw at the ICD connector, lead insulation defect, and lead fracture defined as changes in impedance with changes in sensing/pacing performance (intermittent or permanent) which could be optionally confirmed by X-ray study. Suboptimal and abnormal findings in the device analysis were defined as threshold $>2$ Volts (V) at $0.5 \mathrm{~ms}$, right atrial (RA) lead impedance $>1,500 \Omega$, right ventricular (RV) lead impedance $>2,000 \Omega$, left ventricular (LV) lead impedance $>$ $1,500 \Omega$, RV coil $>200 \Omega$, supraventricular $($ SVC) coil $>200$ $\Omega$, R-wave $<5 \mathrm{mV}$, and $\mathrm{P}$-wave $<0.5 \mathrm{mV}$ [22].

Appropriate therapy consisted of device therapy with ATP and/or shocks for VT or VF. Inappropriate therapy consisted of ATP and/or shocks for heart rhythms other than VT or VF. 


\section{Procedure}

Screening ECG test was performed in two postures (lying down and sitting) for all patients with at least one of the three sensing configurations acceptable in both postures. Two patients failed screening test and underwent TV-ICD implantation.

The procedure was performed in the electrophysiology laboratory under standard sterile conditions and general anesthesia. The left arm was abducted to $60^{\circ}$, and then a dummy of the S-ICD and lead were secured on the patient's chest by adhesive plaster. The positioning of both was guided by anatomical landmarks, as suggested by the manufacturer user's manual [21], with the pocket at the fifth intercostal space between the mid and anterior axillary lines and the lead 1 - 2 $\mathrm{cm}$ to the left of the sternal midline. The position of the lead and S-ICD relative to the heart silhouette was checked by a brief fluoroscopy. Finally, the S-ICD position was drawn onto the chest with a demographic marker pen, as well as the incision line for the pocket creation along the chest Langer's lines. Following sterile draping, an incision was made along the predefined Langer's lines to create the device pocket. Subcutaneous dissection was carried out using an electrosurgical cutting and coagulation device, parallel to the incision, down to the fascia overlying the latissimus dorsi. Intermuscular implantation places the S-ICD device (Cameron Health, SQ RX model 1010 or Boston Scientific, Emblem MRI S-ICD model A219) in the virtual space between the latissimus dorsi and serratus anterior muscles. The pocket was formed over the serratus anterior muscular fascia and beneath the latissimus dorsi muscle by detaching the fibrous tissue between the muscles [22]. The single lead for sensing and defibrillation (Cameron Health Model 3010 Q-TRAK or Emblem S-ICD Electrode model 3401) electrodes positioning was performed following the three- or two-incision techniques [20,21] based on the implanting physician preference. The lead was connected to the generator, and the latter was placed in the pocket. Device analysis was performed to make sure that at least two sensing vectors were acceptable. Non-absorbable sutures were inserted through the connector block suture portal, and a suture knot was tied to anchor the S-ICD to the latissimus dorsi muscle [22].

On the first post-procedure day, the device was analyzed with the patient lying down, standing up and with a short walking distance to choose the best vector.

\section{ICD programming and follow-up}

The conditional shock zone and shock zone were programmed at 190 - 200 and 220 beats per minute, respectively. The patients were followed up initially after 2 months of ICD implant then every 6 months.

\section{Statistical analysis}

Statistical analysis was performed using the SPSS version 20.0 software (IBM Corp., Armonk, NY, USA). Categorical vari- ables were presented in frequency, and differences between the groups were compared with the Chi-square test. Data were presented in mean \pm standard deviation for normally distributed variables or median and interquartile ranges for abnormally distributed variables. Normally distributed variables were compared using independent sample $t$-test. Spearman's correlation analysis was performed to show the relationships between continuous variables. A two-tailed P-value of $<0.05$ was considered statistically significant [23]. Kaplan-Meier time-to-event analyses were conducted with censoring of subjects at their last known status.

\section{Ethical consideration}

This study conformed to the Declaration of Helsinki and its later amendments and was approved by our institution Research Ethics Committee.

\section{Results}

The patients' baseline characteristics are summarized in Table 1 . Thirty patients, 23 males $(76.7 \%)$, age 16 - 64 years with a mean age of $41 \pm 13$ years, received S-ICD for primary prevention in $25(83.3 \%)$ and secondary prevention in five patients $(16.67 \%)$. The indication for ICD implantation was ischemic cardiomyopathy in 11 patients $(36.7 \%)$, non-ischemic cardiomyopathy in 10 patients $(33.3 \%)$, inherited arrhythmia disorders in four patients (13.3\%), and congenital heart disease in five patients $(16.7 \%)$. The follow-up period was 122 1,034 days (mean $710.6 \pm 190$ days). Thirteen patients $(34.3 \%)$ had S-ICD implantation with two-incisions and 17 patients $(56.7 \%)$ with three-incision technique. Two patients $(6.7 \%)$ had wound infection which was treated with antibiotics; one patient $(3.3 \%)$ had a hematoma which was treated conservatively.

During the follow-up, one patient died 22 months after S-ICD implantation due to advanced heart failure. He had ESRD and ischemic cardiomyopathy, and he was on the combined heart and renal transplant list. He had multiple successful shocks for VF episodes. Another patient died with malignancy that was diagnosed 4 months after S-ICD implantation. Four patients received 38 appropriate ICD shocks $(90.5 \%)$, and two patients received four inappropriate shocks $(9.5 \%)$. The first patients with inappropriate shock had congenital heart disease with right bundle branch block and had three inappropriate ICD shocks due to T wave over sensing with physical activity. The change of sensing vector from primary to alternative vector resolved the problem. The second patient also had one shock for T wave oversensing 18 months postS-ICD implant, and it was corrected with reprogramming of the sensing vector.

When compared to 30 consecutive patients (24 males $(80 \%)$; mean age $50 \pm 13.8$ years; mean left ventricular ejection fraction $28 \pm 9.5 \%$ ) who received a single chamber TVICD during the same period, there was no significant difference in mortality. The indication for ICD implantation was 
Table 1. Baseline Clinical Characteristics of the S-ICD and TV-ICD Patients

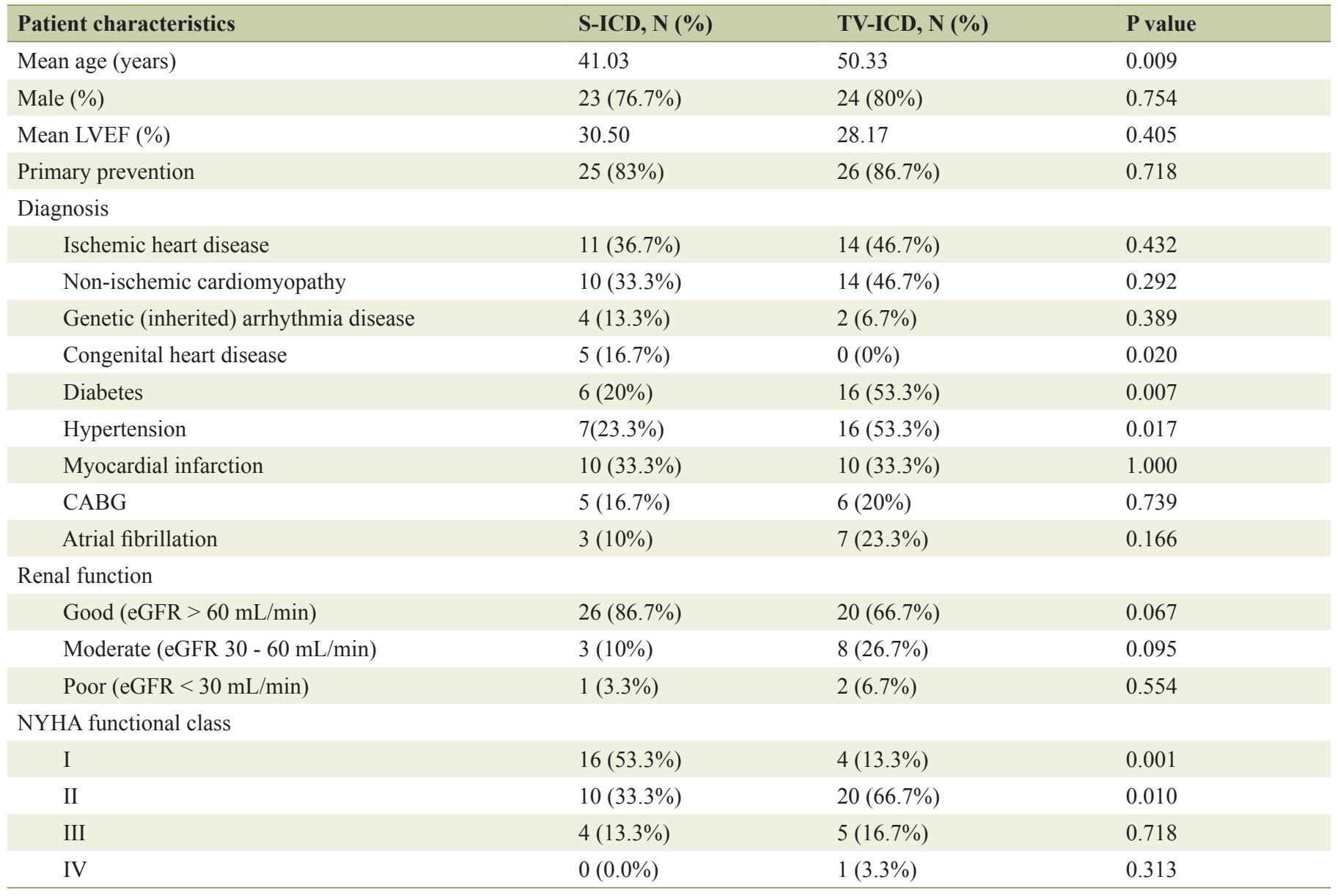

CABG: coronary artery bypass surgery; eGFR: estimated glomerular filtration rate; LVEF: left ventricular ejection fraction; N: number; NYHA: New York Heart Association; S-ICD: subcutaneous implantable cardioverter defibrillators; TV-ICD: transvenous implantable cardioverter defibrillators.

ischemic cardiomyopathy in 14 (46.7\%), non-ischemic cardiomyopathy in 14 (46.7\%), and inherited arrhythmia disorders in two $(6.7 \%)$. There were no patients with congenital heart disease. Two patients $(6.7 \%)$ had a hematoma, which was treated conservatively, and three patients (10\%) needed a second procedure for lead-related problems due to lead dislodgment (one patient), poor sensing and capture with microperforation (one patient) and small $\mathrm{R}$ wave with $\mathrm{T}$ wave oversensing (one patient). The follow-up period was 195 972 days (mean $632 \pm 156$ days). Four patients received 40 appropriate shocks, and one patient received 12 inappropriate shocks. Two patients received 37 appropriate ATP therapies, and one patient received one inappropriate ATP therapy. Overall, the appropriate TV-ICD therapy was $85.6 \%$, and inappropriate therapy $14.4 \%$.

\section{Discussion}

This study suggests that S-ICD implantation without DFT is safe and effective. The outcome was favorable and comparable to TV-ICD. This will be discussed further below regarding the following.

\section{Mortality}

Over an average follow-up of 2 years, the overall mortality in the S-ICD group was $6.7 \%$ and cardiovascular mortality was $3.3 \%$. This is comparable to what has been reported in the pooled analysis of the IDE study and EFFORTLESS registry with an annual mortality rate of $1.6 \%$ and 2 -year mortality rate of $3.2 \%$ [24]. The cardiovascular mortality was similar in the TV-ICD group (Fig. 1). Although the TV-ICD patients were relatively older and had more risk factors for coronary artery disease, the S-ICD group included five patients with complex congenital heart disease and another five patients with prior TV-ICD lead-related infection or fracture. The percentage of secondary prevention cases was not significantly different, $16.7 \%$ in S-ICD group and $13.3 \%$ in the TV-ICD group.

The SCD-Heft study enrolled a mixed population of ischemic and non-ischemic cardiomyopathies and found a risk of death at 2 years in the intervention group of approximately $11-12 \%[25]$. However, low annual mortality rates of $5 \%[26$, 


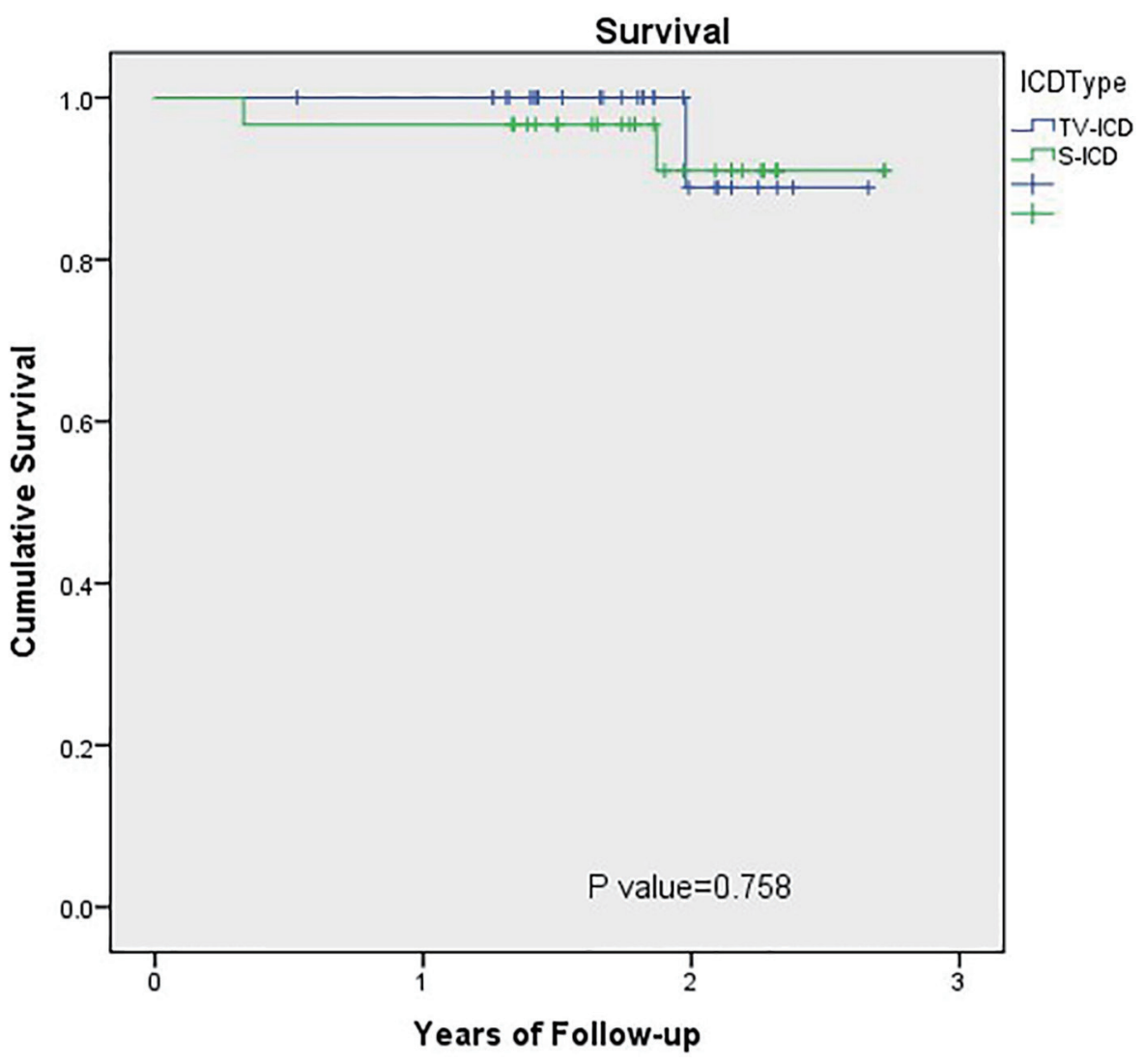

Figure 1. Outcomes comparison of S-ICD and TV-ICD: survival. Kaplan-Meier plot of survival in the S-ICD and TV-ICD patients. S-ICD: subcutaneous implantable cardioverter defibrillators; TV-ICD: transvenous implantable cardioverter defibrillators.

27] and annual appropriate shock rates of 4-5\% [26] have been reported. This is likely secondary to improvements of pharmaceutical and interventional treatment options for coronary artery disease and heart failure [26].

\section{Appropriate S-ICD therapy efficacy}

The S-ICD was effective in terminating all the 42 VT/VF episodes from the first shock. There were no VT/VF episodes that were not detected correctly. The appropriate shocks were $90.5 \%$ of all delivered therapies. For the TV-ICD group, the appropriate TV-ICD therapy (ATP or shock) was $85.6 \%$, and inappropriate therapy was $14.4 \%$. Previous S-ICD studies showed success at the conversion of clinical VT/VF with first shock ranging from $57.9 \%$ to $100 \%[6,24,28-31]$ and from more than one shock from $96.4 \%$ to $100 \%[6,24,28-33]$. In the recent TVICD studies, annual appropriate shock rates were 4-5\% [26].

\section{Inappropriate S-ICD therapy}

Inappropriate therapy occurred in $9.5 \%$ of the S-ICD group and $14.4 \%$ in the TV-ICD group. The incidence of inappropriate shocks was $5-16 \%$ in previous S-ICD studies [6, 24, 28-33]. The cause of inappropriate shock in S-ICD was T- wave oversensing, which is the leading cause of inappropriate shocks in previous S-ICD studies (about $85 \%$ of inappropriate shocks were due to T-wave oversensing) [6, 28].

\section{Device-related complications}

The S-ICD device-related complications were less compared to TV-ICD but not statically significant (Fig. 2). This is consistent with what has been reported before [15]. In the EFFORTLESS registry, the peri-operative complication-free rate in the first 30 days post-implant was 97\% (15 system-related complications occurred in 14 patients) [24]. The documented system or implantation-related complication-free rate was $94 \%$ at 360 days post-implantation [24]. Infection is one of the main complications associated with the S-ICD system. The rate of infection of the generator pocket ranges from $2 \%$ to $10 \%$ in clinical trials, and it may lead to the explanation of the S-ICD system in $1.3-4 \%$ of the patients $[6,24,28-33]$.

\section{Limitations of the study}

The present study is a single-center experience of S-ICD systems without DFT in a small sample size compared with previous multicenter trials with TV-ICD systems. It has the limitation 


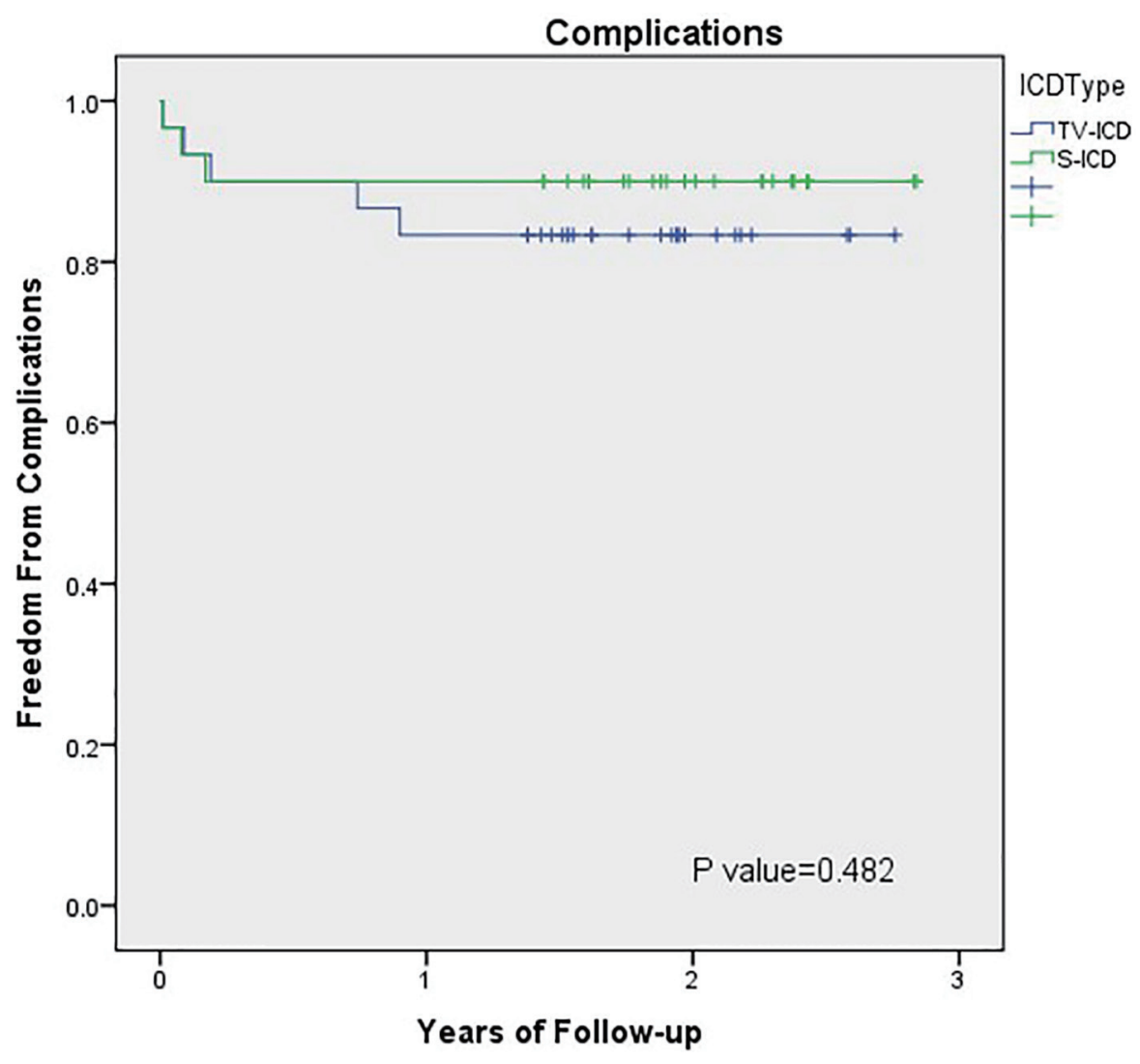

Figure 2. Outcomes comparison of S-ICD and TV-ICD therapy: device-related complications. Kaplan-Meier plot of device-related complications in the S-ICD and TV-ICD patients. S-ICD: subcutaneous implantable cardioverter defibrillators; TV-ICD: transvenous implantable cardioverter defibrillators.

of the retrospective design. All patients in this study underwent S-ICD implantation using intermuscular implantation technique, so the results of this study may not apply to patients who have the S-ICD device implanted using other techniques. Of note, the previous study utilizing the same intermuscular pocket technique showed a successful DFT in all the 14 involved patients [22].

\section{Conclusions}

Implantation of S-ICD with intermuscular approach without DFT seems to be safe and effective. Data from large S-ICD registries with long-term follow-up and ideally randomized controlled studies are needed to confirm this result.

\section{Funding}

This research did not receive any specific grant from funding agencies in the public, commercial, or not-for-profit sectors.

\section{Conflict of Interest}

The authors declare that there is no conflict of interest.

\section{Author Contributions}

All authors certify that he or she has participated sufficiently in the intellectual content, the analysis of data. Each author has reviewed the final version of the manuscript and approves it for publication.

\section{References}

1. Zipes DP, Wellens HJ. Sudden cardiac death. Circulation. 1998;98(21):2334-2351.

2. The Antiarrhythmics versus Implantable Defibrillators (AVID) Investigators. A comparison of antiarrhythmicdrug therapy with implantable defibrillators in patients resuscitated from near-fatal ventricular arrhythmias. N Engl J Med. 1997;337(22):1576-1583.

3. Moss AJ, Hall WJ, Cannom DS, Daubert JP, Higgins SL, Klein H, Levine JH, et al. Improved survival with an implanted defibrillator in patients with coronary disease at high risk for ventricular arrhythmia. Multicenter Automatic Defibrillator Implantation Trial Investigators. N Engl J Med. 1996;335(26):1933-1940.

4. Moss AJ, Zareba W, Hall WJ, Klein H, Wilber DJ, Cannom DS, Daubert JP, et al. Prophylactic implantation of a 
defibrillator in patients with myocardial infarction and reduced ejection fraction. N Engl J Med. 2002;346(12):877883.

5. Bardy GH, Smith WM, Hood MA, Crozier IG, Melton IC, Jordaens L, Theuns D, et al. An entirely subcutaneous implantable cardioverter-defibrillator. N Engl J Med. 2010;363(1):36-44.

6. Weiss R, Knight BP, Gold MR, Leon AR, Herre JM, Hood M, Rashtian M, et al. Safety and efficacy of a totally subcutaneous implantable-cardioverter defibrillator. Circulation. 2013;128(9):944-953.

7. US Food and Drug Administration. News \& Events. FDA approves first subcutaneous heart defibrillator. http:// www.fda.gov/NewsEvents/Newsroom/PressAnnouncements/ucm321755.htm. Published September 28, 2012. Accessed October 13, 2017.

8. Rattes MF, Jones DL, Sharma AD, Klein GJ. Defibrillation threshold: a simple and quantitative estimate of the ability to defibrillate. Pacing Clin Electrophysiol. 1987;10(1 Pt 1):70-77.

9. Singer I, Lang D. Defibrillation threshold: clinical utility and therapeutic implications. Pacing Clin Electrophysiol. 1992;15(6):932-949.

10. Marchlinski FE, Flores B, Miller JM, Gottlieb CD, Hargrove WC, 3rd. Relation of the intraoperative defibrillation threshold to successful postoperative defibrillation with an automatic implantable cardioverter defibrillator. Am J Cardiol. 1988;62(7):393-398.

11. Swerdlow CD, Russo AM, Degroot PJ. The dilemma of ICD implant testing. Pacing Clin Electrophysiol. 2007;30(5):675-700.

12. Russo AM, Chung MK. Is defibrillation testing necessary for implantable transvenous defibrillators?: defibrillation testing is necessary at the time of implantable cardioverter defibrillator implantation. Circ Arrhythm Electrophysiol. 2014;7(2):337-346.

13. Birnie D, Tung S, Simpson C, Crystal E, Exner D, Ayala Paredes FA, Krahn A, et al. Complications associated with defibrillation threshold testing: the Canadian experience. Heart Rhythm. 2008;5(3):387-390.

14. Friedman DJ, Parzynski CS, Varosy PD, Prutkin JM, Patton KK, Mithani A, Russo AM, et al. Trends and in-hospital outcomes associated with adoption of the subcutaneous implantable cardioverter defibrillator in the United States. JAMA Cardiol. 2016;1(8):900-911.

15. Brouwer TF, Yilmaz D, Lindeboom R, Buiten MS, Olde Nordkamp LR, Schalij MJ, Wilde AA, et al. Long-term clinical outcomes of subcutaneous versus transvenous implantable defibrillator therapy. J Am Coll Cardiol. 2016;68(19):2047-2055.

16. Healey JS, Hohnloser SH, Glikson M, Neuzner J, Mabo P, Vinolas X, Kautzner J, et al. Cardioverter defibrillator implantation without induction of ventricular fibrillation: a single-blind, non-inferiority, randomised controlled trial (SIMPLE). Lancet. 2015;385(9970):785-791.

17. Frommeyer G, Zumhagen S, Dechering DG, Larbig R, Bettin M, Loher A, Kobe J, et al. Intraoperative defibrillation testing of subcutaneous implantable cardioverterdefibrillator systems-a simple Issue? J Am Heart Assoc. 2016;5(3):e003181.

18. Wilkoff BL, Fauchier L, Stiles MK, Morillo CA, Al-Khatib SM, Almendral J, Aguinaga L, et al. 2015 HRS/EHRA/ APHRS/SOLAECE expert consensus statement on optimal implantable cardioverter-defibrillator programming and testing. Heart Rhythm. 2016;13(2):e50-86.

19. Russo AM, Stainback RF, Bailey SR, Epstein AE, Heidenreich PA, Jessup M, Kapa S, et al. ACCF/HRS/AHA/ ASE/HFSA/SCAI/SCCT/SCMR 2013 appropriate use criteria for implantable cardioverter-defibrillators and cardiac resynchronization therapy: a report of the American College of Cardiology Foundation appropriate use criteria task force, Heart Rhythm Society, American Heart Association, American Society of Echocardiography, Heart Failure Society of America, Society for Cardiovascular Angiography and Interventions, Society of Cardiovascular Computed Tomography, and Society for Cardiovascular Magnetic Resonance. J Am Coll Cardiol. 2013;61(12):1318-1368.

20. Lewis GF, Gold MR. Clinical experience with subcutaneous implantable cardioverter-defibrillators. Nat Rev Cardiol. 2015;12(7):398-405.

21. Knops RE, Olde Nordkamp LR, de Groot JR, Wilde AA. Two-incision technique for implantation of the subcutaneous implantable cardioverter-defibrillator. Heart Rhythm. 2013;10(8):1240-1243.

22. Ferrari P, Giofre F, De Filippo P. Intermuscular pocket for subcutaneous implantable cardioverter defibrillator: Single-center experience. J Arrhythm. 2016;32(3):223-226.

23. Krousel-Wood MA, Chambers RB, Muntner P. Clinicians' guide to statistics for medical practice and research: part I. Ochsner J. 2006;6(2):68-83.

24. Burke MC, Gold MR, Knight BP, Barr CS, Theuns D, Boersma LVA, Knops RE, et al. Safety and efficacy of the totally subcutaneous implantable defibrillator: 2-year results from a pooled analysis of the IDE study and EFFORTLESS registry. J Am Coll Cardiol. 2015;65(16):16051615.

25. Bardy GH, Lee KL, Mark DB, Poole JE, Packer DL, Boineau R, Domanski M, et al. Amiodarone or an implantable cardioverter-defibrillator for congestive heart failure. N Engl J Med. 2005;352(3):225-237.

26. Seegers J, Conen D, Jung K, Bergau L, Dorenkamp M, Luthje L, Sohns C, et al. Sex difference in appropriate shocks but not mortality during long-term follow-up in patients with implantable cardioverter-defibrillators. Europace. 2016;18(8):1194-1202.

27. Fauchier L, Marijon E, Defaye P, Piot O, Sadoul N, Perier MC, Gras D, et al. Effect of age on survival and causes of death after primary prevention implantable cardioverter-defibrillator implantation. Am J Cardiol. 2015;115(10):1415-1422.

28. Lambiase PD, Barr C, Theuns DA, Knops R, Neuzil P, Johansen JB, Hood M, et al. Worldwide experience with a totally subcutaneous implantable defibrillator: early results from the EFFORTLESS S-ICD Registry. Eur Heart J. 2014;35(25):1657-1665.

29. Olde Nordkamp LR, Dabiri Abkenari L, Boersma LV, Maass AH, de Groot JR, van Oostrom AJ, Theuns DA, 
et al. The entirely subcutaneous implantable cardioverterdefibrillator: initial clinical experience in a large Dutch cohort. J Am Coll Cardiol. 2012;60(19):1933-1939.

30. Jarman JW, Todd DM. United Kingdom national experience of entirely subcutaneous implantable cardioverterdefibrillator technology: important lessons to learn. Europace. 2013;15(8):1158-1165.

31. Aydin A, Hartel F, Schluter M, Butter C, Kobe J, Seifert M, Gosau N, et al. Shock efficacy of subcutaneous implantable cardioverter-defibrillator for prevention of sudden cardiac death: initial multicenter experience. Circ
Arrhythm Electrophysiol. 2012;5(5):913-919.

32. Kobe J, Reinke F, Meyer C, Shin DI, Martens E, Kaab S, Loher A, et al. Implantation and follow-up of totally subcutaneous versus conventional implantable cardioverterdefibrillators: a multicenter case-control study. Heart Rhythm. 2013;10(1):29-36.

33. Dabiri Abkenari L, Theuns DA, Valk SD, Van Belle Y, de Groot NM, Haitsma D, Muskens-Heemskerk A, et al. Clinical experience with a novel subcutaneous implantable defibrillator system in a single center. Clin Res Cardiol. 2011;100(9):737-744. 